\title{
Participation of ABA Metabolism and ROS Generation in Sugar Starvation- Induced Senescence of Rice Flag Leaves
}

\author{
Muhammad Asad Ullah Asad \\ Zhejiang University \\ Fubiao Wang \\ Jinggangshan University \\ Yu Ye \\ Zhejiang University \\ Xianyue Guan \\ Zhejiang University \\ Lujian Zhou \\ Zhejiang University \\ Shamsu Ado Zakari \\ Zhejiang University \\ Zhanyu Han \\ Zhejiang University \\ Fangmin Cheng ( $\square$ chengfm@zju.edu.cn ) \\ Zhejiang University
}

Research article

Keywords: leaf senescence, sugar starvation, ABA, ROS, rice ( Oryza sativa L)

Posted Date: November 21st, 2019

DOI: https://doi.org/10.21203/rs.2.17478/v1

License: @ (i) This work is licensed under a Creative Commons Attribution 4.0 International License. Read Full License 


\begin{abstract}
Background: Both sucrose and abscisic acid (ABA) play pivotal role in the regulation of plant leaf senescence. However, the exact mechanism by which sugar starvation , ABA, and reactive oxygen species (ROS) interact with each other during leaf senescence remains largely unknown. In this study, the genotypedependent alteration in temporal patterns of sugar concentration during leaf senescence and its relation to ABA metabolism and ROS generation were investigated by using the premature senescence of flag leaf ( $p s f$ ) mutant and its wild type.
\end{abstract}

Results: Results showed that sugar starvation-induced leaf senescence was closely associated with the endogenous ABA concentration and ROS level in senescent leaves. Sugar starvation accelerated leaf senescence, concomitantly with the marked increase in ABA concentration and malonaldehyde (MDA) accumulation in detached leaves. Conversely, exogenous sugar treatment significantly suppressed the ABA concentration ad ROS level in detached leaves, thus leaf senescence was delayed by exogenous sugar supply. Pharmacological tests revealed that ABA biosynthesis inhibitor (NDGA) delayed the sugar starvation-induced leaf senescence, while ABA catabolism inhibitor (DNCZ) accelerated leaf senescence and significantly increased the endogenous ABA content in senescent leaves. For the expression patterns of ABA synthesis and catabolism related genes induced by sugar starvation, exogenous sucrose supply, NDGA and DNCZ. sugar starvation up-regulated the OsABA8ox1 transcript, while exogenous sucrose and NDGA down-regulated the transciptional expressions of OsNCED1, OsNCED4 and OsNCED5 and OsABA8ox2 and OsABA8ox3 e by sugar starvation and DNCZ, while the transcript of was increased.

Conclusion: Together, our results demonstrated that the rise in endogenous ABA content during sugar starvation-induced leaf senescence is mostly caused by the suppression of $A B A$ catabolism, rather than the enhancement of $A B A$ biosynthesis, and the expression of $A B A$ metabolic genes determines the equilibrium between $A B A$ biosynthesis and catabolism that eventually influence cross-talk between endogenous factors. The breaking for the equilibrium between $A B A$ biosynthesis and catabolism was strongly responsible for sugar starvation-induced leaf senescence, which was resulted from the suppression of $A B A$ catabolism, rather than the enhancement of ABA biosynthesis .

\title{
Background
}

Leaf senescence is the end of plant developmental phase starts with complex metabolic and physiological changes of chlorophyll (Chl) loss, decreased photosynthetic efficiency, destruction of chloroplast ultra-structures and remobilization of the nutrients, which altogether lead to the death of senescing leaves $[1,2]$. The initiation of destructive metabolic pathways increase lipid peroxidation and membrane leakage, reactive oxygen species (ROS) generation and the hydrolysis of macromolecules, such as proteins, lipids, nucleic acids, and pigments [3,4]. Though leaf senescence develops with the transition of developmental phase (end of vegetative phase) but external stresses (nutrient deficiency, extreme temperatures, drought, heavy mater stress, salt stress and pathogen attack) intervene with endogenous developmental signals [i.e., phytohormones, ROS and sugar levels] to accelerate premature or early leaf senescence which limit grain yield $[5,6]$.

Carbohydrates (sugar) as the integral component of plant metabolism, regulate plant growth, tolerance to external stresses (sugar homeostasis) and productivity. A little deviation in sugar status (sugar starvation) integrate with plant signaling network of hormones to minimize biosynthesis, cellular respiration and cellular growth [7]. The persistence of sugar starvation for long time regulates catabolic activities, oxidative level of cell and nutrient remobilization from vegetative tissues to rice storage grains. Previous studies indicate that dark induced sugar starvation reduce photosynthetic activity, and induce ubiquitin process of chlorophyll degradation [8-11]. The dark induced sugar starvation studies reported the up or down regulation of 800 genes related to the cellular catabolism in Arabidopsis cell suspension cultures [12]. At cellular level, sugar starvation activates a catabolic network responsible for breakdown of starch, lipids, proteins, and other cellular constituents which ultimately damages the cell anabolic function. This sugar starvation induced catabolic activities creates redox imbalance/oxidative stress by excessive ROS accumulation in plant cell. Abscisic acid (ABA) is the prime shield among internal regulatory factors against external stresses and works as a link between oxidative damage of cellular structure and sugar signaling [13-15]. If the extent of sugar starvation and oxidative stress dominates and persist for long time, then ABA regulate the onset of leaf senescence [6]. However, the exact mechanism by which sugar starvation function ABA accumulation and its interaction with ROS accumulation during senescence remains unclear. The trisense loop of sugar starvation, oxidative stress and ABA regulate leaf senescence in complicated manner which is the actual topic of ours debate.

In present study, we used the rice premature senescence of flag leaf ( $p s f)$ mutant and its wild type to assess the genotypic-dependent alteration in sugar, sucrose, hexose, MDA, ROS and ABA contents and their temporal pattern during leaf senescence. Meanwhile, detached leaf segments were employed to in vitro dark treatment, and exogenous sucrose and mannitol treatment, to investigate the effects of artificial sugar starvation on leaf senescence and senescence-related parameters in rice. Moreover, the relationship between sugar starvation and ABA synthesis and catabolism was studied by applying treatments with $A B A$ anabolism inhibitor nordihydroguaiaretic acid (NDGA) and ABA catabolism inhibitor diniconazole (DNCZ) was performed to clarify the link between sugar starvation and ABA metabolism during leaf senescence.

\section{Results}

\section{Genotype-dependent alteration in the senescence-related physiological parameters and their temporal pattern during leaf senescence for the psf mutant and its wild type}

No visible differences between the psf mutant and the wild type were observed at the seedling and early tillering stages. However, leaf senescence symptoms appeared initially on the lower leaves of the psf mutant at the late tillering stage, and the exacerbated lesions subsequently extended to the upper leaves. The flag leaf of psf mutant exhibited senescence symptoms post anthesis, and the lesions first appeared on the leaf tip and then spread gradually downward to cover the whole leaf blade and the leaf sheath. Subsequently, the flag leaf of $p s f$ mutant was completely withered approximately at 30 days post anthesis. By contrast, the leaves in the same position of wild type still remained green during the same period (Fig. 1). The chlorophyll content in the flag leaves of $p s f$ 
mutant dropped earlier than those of its wild type, with more sharply descending pattern for the psf mutant (Fig. 2B). Meanwhile, the MDA accumulation in psf flag leaves increased rapidly after anthesis, but this trend was not observed in the wild type, which showed stable MDA content or a slight increase in MDA content from 0 to 28 days post anthesis (Fig. 2D). Similar to MDA content, the psf mutant had significantly higher $\mathrm{H}_{2} \mathrm{O}_{2}$ concentration in flag leaves than its wild type. Interestingly, the ABA concentrations in the psf leaves increased quickly from 7 days to 28 days post anthesis, whereas those in the wild cultivar increased slightly until rice harvest (Fig. 2l). These results suggested that the genotypic-dependent differences in the timing of senescence initiation and also in the subsequent rate of leaf senescence were closely associated with their varying levels in $A B A$ and $\mathrm{H}_{2} \mathrm{O}_{2}$ contents in rice leaves, because the loss of chlorophyll and oxidative damage of cellular membrane might be caused by the markedly increased levels of ABA and ROS in leaf tissues (Huang et al., 2004; $\mathrm{Li}$ et al., 2018). The marked difference in the temporal pattern of soluble sugar and sucrose contents during leaf senescence was also observed between the two rice genotypes. The psf mutant differed evidently from its wild type in the soluble sugar and sucrose contents and their temporal pattern of during leaf senescence. Comparatively, the soluble sugar and sucrose contents in the flag leaves of psf mutant was significantly lower than those of its WT at the stage of 14 DAA-28 DAA. For the psf mutant, the soluble sugar and sucrose contents in flag leaves decreased continuously with the progression of leaf senescence, while for its WT, soluble sugar and sucrose contents decreased less (Fig. 2E-2G) This result implied that the early initiation and progression of leaf senescence for psf mutant was closely related to lower sugar content and sharply decline in sugar content in the flag leaves.

\section{Linking the sugar starvation-induced leaf senescence with the increasing levels of ABA and ROS}

To clarify the relationship of varying sugar level with $\mathrm{H}_{2} \mathrm{O}_{2}$ and $\mathrm{ABA}$ concentration during leaf senescence, the detached leaf segments of $p s f$ mutant and its wild type were subjected to different incubation period of dark treatments for sugar starvation. Sugar starvation treatment obviously reduced chlorophyll content, but it significantly increased MDA accumulation, $\mathrm{H}_{2} \mathrm{O}_{2}$ and ABA contents in the detached leaves after 3 and 6 days' incubation, regardless of rice genotype with different levels of leaf senescence (Fig. 3). By contrast, the soluble sugar and sucrose contents in leaf segments decreased more markedly with the prolong of sugar starvation treating, although the extent of lowered soluble sugar and sucrose contents in leaf segments was greatly variable, depending on the two rice genotypes (Fig $3 \mathrm{~F}-3 \mathrm{G}$ ). This result indicated clearly that the initiation of leaf senescence might be induced by sugar starvation, in which the increasing levels of $\mathrm{H}_{2} \mathrm{O}_{2}$ and $\mathrm{ABA}$ participated in the induction and acceleration of leaf senescence under sugar starvation.

We further investigated the possible triggering /repressing effect of exogenous sucrose supply on the changing levels of $\mathrm{H}_{2} \mathrm{O}_{2}$ and $\mathrm{ABA}$ by using the detached leaf segments of psf mutant at 0 DAA, with a series of gradient sucrose concentrations $(0 \mathrm{mM}, 40 \mathrm{mM}, 80 \mathrm{mM}, 160 \mathrm{mM}, 320 \mathrm{mM}, 480 \mathrm{mM}, 600 \mathrm{mM})$. The result showed that the exogenous sucrose incubation inhibited the fading of leaf green (Fig. 4A) and significantly enhanced the chlorophyll content in detached leaves (Fig. 4B). By contrast, the detached leaf segments faded quickly along with decline in chlorophyll content when the similar concentrations of mannitol were supplied to sugar starvation-induced leaf senescence (Fig. 4A-4B). Interestingly, exogenous sucrose incubation sharply decreased the $\mathrm{H}_{2} \mathrm{O}_{2}$ content and $\mathrm{O}_{2}{ }^{-}$generation in detached leaves (Fig. 4C-4D), and the ABA concentration and MDA content in the detached leaf segments treated by $>80$ mM exogenous sucrose concentration were significantly lower than those incubated in mannitol solution (Fig. 4C and 4F). These results implied that the contribution of exogenous sucrose incubation to the delayed leaf senescence in detached segments was closely associated with the repressing effect of exogenous sucrose supply on the ROS level and ABA concentration in detached segments.

\section{Genotype-dependent differences in the transcriptional profile and the temporal patterns of key genes involving in ABA biosynthesis and catabolism during leaf senescence}

The transcriptional profiles of key genes participating inABA biosynthesis and catabolism (OsNCED and OsABA80x) and genotype-dependent changes in their temporal patterns during leaf senescence were comprehensively investigated by quantitative real-time reverse transcription PCR (Fig. 5). The differential expression of five ABA synthetic genes (OsNCED1-5) and three ABA catabolic genes (OsABA8ox1-3) in various organs was also detected to elucidate their spatial patterns in rice plants. The OsNCED1, OsNCED4, OsNCED5, OsABA80x1, OsABA80x2 and OsABA80x3 genes expressed preferentially in rice leaves, with relatively low transcription or undetectable levels in culm and grain (Fig. $5 \mathrm{H})$. OsNCED1, OsABA8ox2 and OsABA8ox3 genes were highly expressed in the leaves and sheaths and moderately expressed in the culm and grains, compared to those in the psf mutant, these genes displayed higher expression in wild type. OsNCED5 gene was only detectable expressed in the leaf, whereas OsNCED2 was only lowly expressed in the culm in the psf mutant and its wild type, and OSABA80x1 was lowly expressed in various organs, with slight difference between the two genotypes. Thus, OsNCED1 and OsABA80x3 expressions were more closely related to the alteration of $A B A$ levels in the genotype-dependent senescence than the other ABA metabolic regulatory genes during sugar starvation-induced leaf senescence.

For five OSNCEDs and three OSABA80x isoforms, OsNCED1 and OSABA80x3 were highly expressed in rice leaves, and the transcriptional levels of OsNCED4, OsNCED5, OsABA80x1, and OSABA80x2, were moderately or lowly abundant for two rice genotypes, whereas the transcripts of OsNCED2 and OsNCED3 were undetectable due to an extremely low level oftranscriptional expression (Fig. $5 \mathrm{H}$ ). Comparatively, the psf mutant generally had remarkably lower transcripts of OsNCED1 and OSABA80X3 than its wild type, with the reducing trend along with leaf senescence (Fig. 5B and 5G). This result implied that the down-regulation of OSNCED1 and OSABA8Ox3 participated in the regulation of ABA concentration in psf mutant leaves, which might be closely associated with the genotypedependent alteration in the invitation and progression of leaf senescence. Considering the strongly elevated $A B A$ concentration during leaf senescence and the genotype-dependent alteration in leaf senescence process, we presumed that the strikingly increased ABA concentration in the senescent leaves of $p s f$ mutant was more attributable to the suppression of ABA catabolism, rather than the enhancement of ABA biosynthesis during leaf senescence. 
To elucidate the relationship of ABA biosynthesis and catabolism with sugar starvation-induced leaf senescence, we further examined the transcripts of several genes involving in ABA biosynthesis and catabolism by using the detached leaf segments under darkness. The result showed that the transcripts of OsNCED 1, OsNCED4, and OSABA80x3 in psf mutant leaves was more severely suppressed than its wild after 6 day's darkness. OsNCED4 expression in psf mutant leaf was downregulated by approximately 5 -fold after 6 day dark treatment, whereas only 0.5 -fold decrease was observed for wild type at the same incubation stage (Fig. 6). By contrast, the transcript of OSABA80x 1 was significantly enhanced by sugar starvation treatment after 3 day and 6 day incubation under darkness (Fig. 6D). These results further indicated that the increasing levels of $A B A$ in sugar starvation-induced leaf senescence were mainly attributable to the weakening of $A B A$ catabolism, as reflected by the down-regulation of OsABA80x3 transcript, rather than the enhancement of $A B A$ biosynthesis.

The transcripts of OSNCED1 and OSABA80x3 involving in ABA biosynthesis and catabolism in response to the varying sugar concentration was further investigated by the detached leaf segments floated in different exogenous sucrose concentration (Fig. 7). Transcripts of OsNCED1, OsNCED4, and OsABA8ox3 were significantly induced by the incubations of higher sucrose concentration ( $>80 \mathrm{mM})$. In term of the increasing extent, the $O s A B A 80 x 3$ isoform displayed the highest increase among the four isoform genes, upregulated by approximately 2-fold, whereas only 0.8-fold increase was observed for OsNCED1 expression under the same sucrose treatment $(80 \mathrm{mM})$ (Fig 7F and Fig 7A). The transcriptional patterns of ABA metabolic genes were consistent with the decreasing patterns of ABA content for higher levels of sucrose treatment $(>80 \mathrm{mM})$. These results suggested that the inhibition of leaf senescence by exogenous sugar was closely related to the enhancement of ABA catabolism, thereby inhibiting the increase of ABA content in detached leaf segments under exogenous sucrose treatment.

\section{Involvement of ABA catabolism and ABA metabolism with dark induced leaf senescence}

To detect whether endogenous ABA was the inducer of sugar starvation-induced leaf senescence, ABA biosynthesis and catabolism inhibitors, NDGA and DNCZ, were applied. As shown in Fig. 8, compare with the control treatment, the accumulation of endogenous ABA was effectively retarded by the ABA biosynthesis inhibitor, while the ABA catabolism inhibitor was just the opposite, significantly induced the accumulations of $A B A$. The variations of the fading leaf green, lowering chlorophyll content, $\mathrm{H}_{2} \mathrm{O}_{2}$ and MDA accumulations, soluble sugar, and sucrose content were consistent with the endogenous $\mathrm{ABA}$ content in inhibitors and control samples. The NDGA incubation significantly delayed sugar starvation-induced leaf senescence, increased the chlorophyll, soluble sugar, and sucrose contents, decreased the $\mathrm{H}_{2} \mathrm{O}_{2}$ and MDA accumulations. On other hand, DNCZ treatment accelerated the fading green symptoms, reduced the contents of chlorophyll, soluble sugar, and sucrose content, and significantly increased the $\mathrm{H}_{2} \mathrm{O}_{2}$ and MDA accumulations. These results demonstrated that the sugar starvation-induced leaf senescence dependent on the endogenous $A B A$ accumulation. And the rising levels of endogenous $A B A$ in sugar starvation-induced leaf senescence was a result of the suppression of ABA catabolism rather than the enhancement of ABA biosynthesis

\section{Discussion}

Sucrose is the main end-product of photosynthesis, used for the transport and redistribution of photosynthetic products in higher plants [16]. The little deviation of sugar due to environmental stresses affect sugar signaling and mediate regulatory effect of sugar on the leaf senescence [8, 17]. For instance, the endogenous sugar concentrations markedly decreased during drought induced leaf senescence in sorghum [9]. Our study found the reduced soluble sugar, sucrose and hexose in senescent flag leaves of psf mutant. The darkness treatment of detached leaf segments of the psf mutant and its wild type induce leaf senescence. Contrarily to our findings, One et al. (1996) found that endogenous sugar contents of sunflower increased leaf senescence in low N. In contrast to sugar accumulation hypothesis [18], high sucrose concentration by $>160 \mathrm{mM}$ delayed the onset of leaf senescence in detached leaf segments in our study. These observations are supported by previous findings of Fujiki et al. [19] and Xiao et al. [20] in Arabidopsis. The imbalance of sugar and sucrose ratios in senescent leaves has been accredited in previous studies on sugars starvation-induced leaf senescence [21]. In this study, we found that the decline of hexose was significantly less than that of sucrose in the early stage (from 0 day to 7 DAA) of flag leaf senescence in $p s f$ mutant. The sugar starvation treatment of detached leaf segments also exhibited similar trend (Fig. $3 \mathrm{H}$ ). This early decline of hexose content in the psf leaf (at 0-day post anthesis) may have accelerated the unloading of sucrose from the leaf and its decomposition into hexose, and a result the changing rate of hexose to sucrose was increased. Therefore, the increased fluctuating ratio of hexose and sucrose greatly contribute to the onset and acceleration of sugar starvation induced leaf senescence during the grain filling stage. Low cell-wall invertase activity by INVINH1 gene repression reduces the apoplasmic hexose level that eventually promote the transport of sucrose from leaf carbohydrate storage to phloem, and thus reducing carbohydrate level in leaves which cause leaf senescence.

ABA being the most important stress responsive plant hormone is involved in regulation of many physiological and developmental processes, such as stomatal closure during drought, dormancy of seeds under extreme environmental conditions and leaf senescence due to internal and external factors [6]. Research involving physiological and genetic analysis have affirmed a positive correlation between sugar and ABA signaling [22, 23]. Arabidopsis mutants with decreased sugar sensitivity are either ABA insensitive (abi mutant) or ABA deficient (aba mutants) during the early stages of plant development. Dark treatment with reduced sugar contents in Arabidopsis seedling and cell suspension cultures has increased ABA contents [24, 25]. Rolland et al. [26] found that sugar starvation in Arabidopsis could promote endogenous ABA level and ABA sensitivity. Likewise, we also found a clear correlation between decreased sugar content and increased endogenous ABA content in our sugar starvation treatments. Previous studies reported that exogenous ABA induce leaf senescence in wheat flag leaves by inducing the expression of many senescence-associated genes (Yang et al. [27] [28]. The endogenous level of ABA in plant tissues was controlled by the equilibrium between ABA biosynthesis and catabolism. Arabidopsis sugar-insensitive mutants expressed exacerbate endogenous $A B A$ levels and delayed leaf senescence conferring that sugar signaling regulate $A B A$ to induce/delay leaf senescence, while increased $A B A$ content was found in early leaf senescent mutant $[29,30]$. ABA acts as bridge between sugar starvation and leaf senescence as ABA-deficient mutants showed accelerated senescence on glucose-containing medium [31]. (move it to discussion section!). 
Moreover, the sugar starvation induced leaf senescence has been reported to possibly promote of $\mathrm{H}_{2} \mathrm{O}_{2}$ accumulation in rice [32-34]. The decreased sugar contents increased electron transport ratio and created oxidative stress which consequently increased ROS level under sugar starvation. The energy production (ATP) keep the balance of ROS in cells and sugar starvation reduced ATP level let to increase ROS in the cells leading cell damage; leaf senescence [35]. During the process of leaf senescence, regulatory switch of ABA may induce ROS accumulation under sugar starvation. Under field condition, Psf mutant also show increased ROS level along with leaf senescence symptoms. Under sugar starvation, the ABA content in leaf of the $p s f$ mutant was significantly higher along with increased ROS with the aggravation of leaf senescence. Moreover, supplementing exogenous sugar to the detached leaves inhibited the increase of ABA content and ROS level, and subsequently inhibited the process of senescence. Our results are corroborated by the findings of Ruan et al. [21] where the INVINH1 gene inhibitor suppressed the degradation of endogenous sugar that resulted into the suppression of ABA, ROS accumulation, expression of senescence-related genes which altogether suppresses leaf senescence. Further studies are required to investigate the causal relationship between these three factors (sugar, ABA and ROS) in regulating leaf senescence and their signal interaction mechanism by silencing the related gene.

Sugar starvation may induce ABA accumulation by regulating ABA biosynthesis and suppressing ABA catabolism. Previous studies has reported an equilibrium between $A B A$ biosynthesis and $A B A$ catabolism during stress induced leaf senescence [36-38]. Meanwhile, our analysis demonstrated that rise of $A B A$ content was due to reduced ABA catabolism under sugar starvation induced leaf senescence. The suppression of ABA catabolism by DNCZ increased ABA contents, while suppression of ABA biosynthesis by NDGA decreased ABA contents in sugar starved detached leaf segments, thereby DNCZ induced leaf senescence and NDGA suppresses leaf senescence in dark induced sugar starvation. Our finding accredits ABA catabolism as a limiting factor for $A B A$ accumulation and ABA induced leaf senescence. Moreover, NDGA suppression of ABA biosynthesis and leaf senescence in dark induced sugar starved leaves indicates that sugar starvation induces leaf senescence through ABA mediated pathway. We further used RT-qPCR to determine the expression patterns of ABA metabolism-related genes (OsNCED for ABA biosynthesis and OsABA8ox for ABA catabolism) in flag leaves of the psf mutant and its wild type after anthesis (i.e., natural senescence during grain-filling), and detached leaf segments subjected to sugar starvation, exogenous sucrose and NDGA/DNCZ. Down regulation of ABA biosynthesis genes (OsNCED1, OsNCED4 and OsNCED5) and upregulation (up to many fold) of OsABA8Ox1 accumulatively increased ABA contents in sugar starvation induced leaf senescence. It is clear that the increasing $A B A$ contents during sugar starvation-induced leaf senescence was not due to higher expression of the ABA biosynthetic regulatory genes, but could be due to inhibition of the ABA catabolism genes (OsABA8ox1, OsABA8ox2 and OSABA80x3).『Similar responses of differential expression of OsNCED and OSABA8ox genes, and varying ABA content has been reported during rice seed germination responses induced by stress $[39,40]$. Our results also indicated that the ABA contents notably decreased with the increasing levels of sucrose treatment, where the expressions of $0 s A B A 80 \times 2$ and $0 s A B A 80 \times 3$ genes were significantly up-regulated. $\square$ We assume that the decrease in $A B A$ contents during delayed leaf senescence mainly depends upon its increased catabolism, whereas the up-regulated expression of its biosynthesis genes may have resulted from the feedback regulation of the decreased ABA level. Interestingly, the transcription of OSNCED genes exhibited significant up-regulation rather than downregulation during NDGA incubation, which may be caused by ABA feedback modulation. However, the ABA catabolism inhibitor (DNCZ) dramatically suppressed the expression of ABA catabolic genes, which increased ABA content and induced leaf senescence respectively. Our observations are supported by the findings of Mega et al. [41], and it can be deduced that the rising ABA contents during sugar starvation-induced leaf senescence relies on the downregulation of $A B A$ catabolic genes among which the differential expression of OsABA80x3 may play a decisive role.

\section{Conclusion}

This study provides clear insight into the mechanisms of ABA dependent senescence in rice leaves induced by sugar content. The synchronization cross talk between sugar starvation, $A B A$ and leaf senescence clarified that sugar starvation induces leaf senescence by rapidly decreasing the soluble sugar, sucrose and hexose level, and subsequent increase in ABA. Moreover, increasing the sucrose content (exogenous) gradually decreases the ABA level and leads to delay the process of leaf senescence. Hence, sugar content (sucrose, fructose and hexose) reciprocally regulate the content of $A B A$, once the $A B A$ anabolism is stopped (by exogenous NDGA) or delayed by external or internal stimuli, leaf senescence is delayed irrespective of the sugar content. The inhibition or decrease of $A B A$ catabolism than $A B A$ anabolism is the rate limiting step of leaf senescence. Our investigation confers the $A B A$ as an inducing agent, a connecting bridge between sugar and leaf senescence. The fluorescence quantitative PCR made it clear that the equilibrium between ABA synthesis and catabolism is maintained by the regulation of OSNCED1 and OSABA80x3 genes, and ABA catabolism basically responsible for the onset of leaf senescence in rice. However, further studies, involving by modern molecular techniques of gene silencing and/or over expression of ABA catabolism related genes, are required to elucidate the relationship between $A B A$ metabolism, sugar starvation and ROS during leaf senescence.

\section{Materials And Methods Plant materials and field experiment}

Two rice genotypes, an indica rice cultivar (Zhehui7954) and its corresponding mutant with the psf (premature senescence of flag leaf mutant after anthesis) phenotype were used in this study. The psf mutant was derived from Zhehui7954 (Oryza sativa L.ssp. indica) by gamma-irradiated Zhehui7954 mature seeds, and the stably inherited mutant was obtained through the successive self-pollination and their identifications of plant phenotype in M2-M7 generations. The field experiments were conducted in 2016 and 2017 at the experimental station of Zhejiang University $\left(120^{\circ} 04^{\prime} \mathrm{E}, 3^{\circ}{ }^{\circ} 18^{\prime} \mathrm{N}\right)$, Hangzhou, China. Rice seeds were sown in April 21 and transplanted in May 20. The field plots were arranged by following a randomized block design with three replicates for each genotype. Each replication was planted in $10 \times 12$ rows, and plant spacing was $18 \mathrm{~cm} \times 18 \mathrm{~cm}$ with one rice seedling for each hill. The field management was carried out according to the local practices. The soil type was the periodically waterlogged paddy soil, with $1.71 \mathrm{~g} / \mathrm{kg}$ of total N, $23.8 \mathrm{mg} / \mathrm{kg}$ of available P and 114.2 $\mathrm{mg} / \mathrm{kg}$ of exchangeable $\mathrm{K}$. At the full heading day, a total of 70-80 plants with uniform anthesis were randomly selected and tagged.

The rice plants were sampled at grain filling stage. 50-70 panicles with uniformity anthesis day were randomly selected and tagged at the full heading day. The flag leaves corresponding to the tagged panicles were sampled from the full heading stage to the mature stage with a 7-day interval. Leaf samples were 


\section{Exogenous incubation experiment in detached leaves}

Experiment l: To investigate the association of sugar starvation mediated leaf senescence with the ROS generation and ABA concentration in the senescing leaves, the flag leaves of the psf mutant and its wild type were carefully detached from plants at 14 days after anthesis (DAA). Detached leaves of both genotypes were cut into $3 \mathrm{~cm}$ and soaked in petri dishes containing sterilized distilled water. For sugar starvation treatment, these petri dishes were placed in artificial growth chamber (Model PRX-450D; Safu, Shanghai, China) at $25^{\circ} \mathrm{C}$ under complete darkness, and the leaf segments were subsequently sampled at a 3-day interval (0-6 day after the initial incubation). All detached leaf samples were kept frozen and then stored at $-80^{\circ} \mathrm{C}$ after an observation for its senescence symptoms. Three replications (petri dishes) were performed for each sampling time.

Experiment II: The effect of exogenous sugar concentration on the transcriptional expressions of various genes involving in ABA biosynthesis and its catabolism was further examined by using the detached leaf segments of the $p s f$ mutant. Detached leaf segments were imposed to different concentration ABA treatments, the gradient concentration of exogenous sucrose was $0 \mathrm{mM}, 40 \mathrm{mM}, 80 \mathrm{mM}, 160 \mathrm{mM}, 320 \mathrm{mM}$ and $600 \mathrm{mM}$, respectively. For each treatment, $25 \mathrm{ml}$ of sucrose solution was added in Petri dishes, with 4 dishes for each incubating concentration. The similar incubation with a series of mannitol concentration was used as control. Prior to these immersions, the leaf segments were placed in distilled water for $2 \mathrm{~h}$ to eliminate the wound stress. After 6 days-incubation at $25^{\circ} \mathrm{C}$ under complete darkness, the detached leaf segments were sampled and stored for subsequent analysis after their senescence symptoms being recorded with a digital camera.

Experiment III: To verify the contribution of ABA metabolism in the senescing leaves to the regulation of sugar starvation induced leaf senescence, the detached leaf segments of the psf mutant at 14 DAA were employed to conduct an experiment on the artificially induced alterations in endogenous ABA concentration and its metabolism for the senescing leaves. In this experiment, the detached leaf segments were soaked in Petri dishes containing $25 \mathrm{ml}$ solution of $10 \mathrm{mM}$ nordihydroguaiaretic acid (NDGA, an inhibitor for plant ABA biosynthesis) and $10 \mathrm{mM}$ diniconazole (DNCZ, an inhibitor for plant ABA catabolism), respectively. The similar incubation with distilled water was implemented as control. 12 replications were performed for each treatment. All detached leaves were incubated at $25^{\circ} \mathrm{C}$ under complete darkness, the leaf segments were subsequently sampled at 0 day, 3 days and 6 days after the initial incubation. The visual symptoms of leaf senescence for different treatments were recorded with a digital camera, and the sampled leaf segments were kept at $-80^{\circ} \mathrm{C}$ until further analysis.

\section{Measurement of chlorophyll, soluble sugar, sucrose, superoxideradical $\left(\mathrm{O}_{2}{ }^{-{ }^{-}}\right)$, hydrogen peroxide $\left(\mathrm{H}_{2} \mathrm{O}_{2}\right)$, and malondialdehyde (MDA) contents in rice leaves}

The chlorophyll content in rice leaves was determined spectrophotometrically as described by Lichtenthaler (1987), using a Shimadzu UV-vis $2450 / 2550$ spectrophotometer. Soluble sugar and sucrose contents were assayed according to the method described previously by Yang et al. (2003). Superoxide radical $\left(\mathrm{O}_{2}{ }^{\cdot-}\right)$ generation rate, hydrogen peroxide $\left(\mathrm{H}_{2} \mathrm{O}_{2}\right)$, and malondialdehyde (MDA) contents were measured by following the procedure as described by Rao et al. [42]. For all these parameters described above, the flag leaves and/or its detached leaf segments were used for each measurement, with 3-4 biological replicates.

\section{Extraction and determination of ABA in rice leaves}

The extraction and purification of ABA in rice flag leaves was carried out according to the method of Kojima et al. [43] with some modifications. One-gram aliquot of fresh leaf samples was homogenized in liquid nitrogen and added to $5 \mathrm{ml}$ of frozen extraction buffer (methanol: formic acid: water = 15: 1: 4). After $24 \mathrm{~h}$ extraction at $-20^{\circ} \mathrm{C}$, the homogenate was centrifuged at $10,000 \times \mathrm{g}$ for $15 \mathrm{~min}$ at $4{ }^{\circ} \mathrm{C}$. The supernatants from each sample were transferred to a 96 -well collection plate and the remaining residues were extracted again. Subsequently, the collection plate containing the sample was placed in the automatic extraction of solid phase extraction system (SPE215; Gilson, Middleton, WI, USA). After extraction, the residual extraction was activated with 1 mol L ${ }^{-1}$ formic acid before through column. The ABA was washed out using methanol. and the eluent was evaporated and further purified. Afterward, ABA content was measured by UPLC-ESI-qMS/MS method. The chromatographic conditions and MS parameters in UPLC-ESI-qMS/MS system were designed as described by Kojima et al. (2009). Three replications were detected for each sampling.

\section{RNA isolation, cDNA preparation, and Quantitative real-time PCR}

The procedures of RNA extraction and cDNA preparation for leaf tissues were performed as described by Wang et al. (2016). Trizol reagent kit (Invitrogen, Carlsbad, CA, USA) was used for the extraction of total RNA, the First Stand cDNA Synthesis kit (Toyobo, Osaka, Japan) was used for cDNA synthesis by following the manufacturer instructions in manual. Quantitative real-time PCR was performed by using the SYBR Green real-time PCR Master Mix Reagent Kit (Toyobo, Osaka, Japan). The reactions were performed in Bio-Rad CFX96 real-time system (Bio-Rad, CA, USA) by following the manufacturer's protocol. The amplification reagents contained $10 \mu \mathrm{L}$ SYBR, $1 \mu \mathrm{L}$ cDNA, $1.6 \mu \mathrm{L} 10 \mathrm{mM}$ primer pairs and $7.4 \mu \mathrm{L}$ RNase Free $\mathrm{H}_{2} \mathrm{O}$. All gene-specific primer pairs were designed by using online software GenScript, and the optimal primer of annealing temperature for each gene used in this study was listed in supplementary Table S1. The expression of Actin was used as internal control. The amplification of various genes was normalized by $A C T I N-1$ expression and their relative expression levels were calculatedby $2^{(-\Delta \Delta C T)}$ method $[44,45]$. Each experiment was performed with three replicates. 


\section{Statistical analysis}

All determinations were performed in at least three independent experiments. Statistical differences were analyzed by analysis of variance (ANOVA) using the SPSS statistical software package (Chicago, Illinois, USA). The mean were compared by the least significant difference (LSD) test at probability level of $p<$ 0.05 .

\section{Declarations}

\section{Funding}

The authors are deeply indebted to National Key Research and Development Plan of China (No 2016YFD0300502 and 2017YFD0300103) and National Natura

\section{Ethic approval and consent to participate}

Not applicable

\section{Consent for publication}

Not applicable

\section{Availability of data and materials}

All data and materials generated or analyzed during this study are included in this article or are available from the corresponding author on reasonable request

\section{Competing interests}

The authors declared that they have no competing interest.

\section{Acknowledgements}

The authors are deeply grateful to Dr. Gan Pan for careful review of manuscript.

\section{Authors' contributions}

Conceived and designed the experiments: MAU, FW and FC. Performed the experiments: MAU FW and YY. Analyzed the data: MAU, FW, XG, SAZ and ZH. Wrote the paper: MAU, FW and FC. All of the authors reviewed the manuscript.

All authors read and approved the final manuscript.

\section{Availability of data and materials}

The datasets supporting the results of this article are included with in the manuscript.

\section{Ethics approval and consent to participate}

Not applicable

\section{Consent for publication}

Not applicable.

\section{Competing interests}

The authors declare that they have no competing interests.

\section{Author details}

${ }^{1}$ Institute of Crop Science, College of Agriculture and Biotechnology, Zhejiang University, Hangzhou 310058, China

2 Jiangsu Collaborative Innovation Centre for Modern Crop Production, Nanjing, China

${ }^{3}$ Research Center for Jinggangshan Eco-Environmental Sciences, Jinggangshan

University, Ji'an 343009,

\section{References}

1. Fischer AM: The Complex Regulation of Senescence. Critical Reviews in Plant Sciences 2012, 31(2):124-147. 
2. Gan S, Amasino RM: Making sense of senescence (molecular genetic regulation and manipulation of leaf senescence). Plant physiology 1997 , 113(2):313.

3. Woo HR, Kim HJ, Nam HG, Lim PO: Plant leaf senescence and death-regulation by multiple layers of control and implications for aging in general. J Cell Sci 2013:jcs. 109116.

4. Watanabe M, Balazadeh S, Tohge T, Erban A, Giavalisco P, Kopka J, Mueller-Roeber B, Fernie AR, Hoefgen R: Comprehensive dissection of spatio-temporal metabolic shifts in primary, secondary and lipid metabolism during developmental senescence in Arabidopsis thaliana. Plant physiology 2013:pp. 113.217380 .

5. Lim PO, Woo HR, Nam HG: Molecular genetics of leaf senescence in Arabidopsis. Trends in plant science 2003, 8(6):272-278.

6. Asad MAU, Zakari SA, Zhao Q, Zhou L, Ye Y, Cheng F: Abiotic Stresses Intervene with ABA Signaling to Induce Destructive Metabolic Pathways Leading to Death: Premature Leaf Senescence in Plants. International Journal of Molecular Sciences 2019, 20(2):256.

7. Chen Y-S, Ho T-HD, Liu L, Lee DH, Lee C-H, Chen Y-R, Lin S-Y, Lu C-A, Yu S-M: Sugar starvation-regulated MYBS2 and 14-3-3 protein interactions enhance plant growth, stress tolerance, and grain weight in rice. Proceedings of the National Academy of Sciences 2019, 116(43):21925-21935.

8. Wingler A, Purdy S, MacLean JA, Pourtau N: The role of sugars in integrating environmental signals during the regulation of leaf senescence. Journal of experimental botany 2005, 57(2):391-399.

9. Chen D, Wang S, Xiong B, Cao B, Deng X: Carbon/nitrogen imbalance associated with drought-induced leaf senescence in Sorghum bicolor. PloS one 2015, 10(8):e0137026.

10. Thimann KV, Tetley RM, Krivak BM: Metabolism of oat leaves during senescence: V. Senescence in light. Plant Physiology 1977, 59(3):448-454.

11. Shi H, Wang B, Yang P, Li Y, Miao F: Differences in sugar accumulation and mobilization between sequential and non-sequential senescence wheat cultivars under natural and drought conditions. PloS one 2016, 11(11):e0166155.

12. Buchanan-Wollaston V, Page T, Harrison E, Breeze E, Lim PO, Nam HG, Lin JF, Wu SH, Swidzinski J, Ishizaki K: Comparative transcriptome analysis reveals significant differences in gene expression and signalling pathways between developmental and dark/starvation-induced senescence in Arabidopsis. The Plant Journal 2005, 42(4):567-585.

13. Zhao Y, Chan Z, Gao J, Xing L, Cao M, Yu C, Hu Y, You J, Shi H, Zhu Y: ABA receptor PYL9 promotes drought resistance and leaf senescence. Proceedings of the National Academy of Sciences 2016, 113(7):1949-1954.

14. Lee IC, Hong SW, Whang SS, Lim PO, Nam HG, Koo JC: Age-dependent action of an ABA-inducible receptor kinase, RPK1, as a positive regulator of senescence in Arabidopsis leaves. Plant and Cell physiology 2011, 52(4):651-662.

15. Yang J, Worley E, Udvardi M: A NAP-AAO3 regulatory module promotes chlorophyll degradation via ABA biosynthesis in Arabidopsis leaves. The Plant Cell 2014:tpc. 114.133769.

16. Fan J, Chen C, Brlansky R, Gmitter Jr F, Li ZG: Changes in carbohydrate metabolism in Citrus sinensis infected with 'Candidatus Liberibacter asiaticus'. Plant Pathology 2010, 59(6):1037-1043.

17. Wingler A, Roitsch T: Metabolic regulation of leaf senescence: interactions of sugar signalling with biotic and abiotic stress responses. Plant Biology 2008, 10:50-62.

18. Quirino BF, Noh Y-S, Himelblau E, Amasino RM: Molecular aspects of leaf senescence. Trends in plant science 2000, 5(7):278-282.

19. Fujiki Y, Yoshikawa Y, Sato T, Inada N, Ito M, Nishida I, Watanabe A: Dark-inducible genes from Arabidopsisthaliana are associated with leaf senescence and repressed by sugars. Physiologia Plantarum 2001, 111(3):345-352.

20. Xiao S, Gao W, Chen Q-F, Chan S-W, Zheng S-X, Ma J, Wang M, Welti R, Chye M-L: Overexpression of Arabidopsis acyl-CoA binding protein ACBP3 promotes starvation-induced and agedependent leaf senescence. The Plant Cel/ 2010:tpc. 110.075333.

21. Ruan Y-L, Jin Y, Huang J: Capping invertase activity by its inhibitor: roles and implications in sugar signaling, carbon allocation, senescence and evolution. Plant signaling \& behavior 2009, 4(10):983-985.

22. Gazzarrini S, McCourt P: Genetic interactions between ABA, ethylene and sugar signaling pathways. Current opinion in plant biology 2001, 4(5):387-391.

23. Han C-s, Kim S, Lee S-e, Choi S, Kim S-h, sun Yoon I, Hwang Y-s: Cross-talk between ABA and sugar signaling is mediated by the ACGT core and CE1 element reciprocally in OsTIP3; 1 promoter. Journal of plant physiology 2018, 224:103-111.

24. Fujii $\mathrm{H}$, Zhu J-K: Arabidopsis mutant deficient in 3 abscisic acid-activated protein kinases reveals critical roles in growth, reproduction, and stress. Proceedings of the National Academy of Sciences 2009, 106(20):8380-8385.

25. Liebsch D, Keech O: Dark-induced leaf senescence: new insights into a complex light-dependent regulatory pathway. New Phytologist 2016, 212(3):563570.

26. Rolland F, Moore B, Sheen J: Sugar sensing and signaling in plants. The plant cel/ 2002, 14(suppl 1):S185-S205.

27. Yang J, Zhang J, Wang Z, Zhu Q, Liu L: Involvement of abscisic acid and cytokinins in the senescence and remobilization of carbon reserves in wheat subjected to water stress during grain filling. Plant, Cell \& Environment 2003, 26(10):1621-1631.

28. Parkash J, Vaidya T, Kirti S, Dutt S: Translation initiation factor 5A in Picrorhiza is up-regulated during leaf senescence and in response to abscisic acid. Gene 2014, 542(1):1-7.

29. Song Y, Xiang F, Zhang G, Miao Y, Miao C, Song C-P: Abscisic acid as an internal integrator of multiple physiological processes modulates leaf senescence onset in Arabidopsis thaliana. Frontiers in plant science 2016, 7:181

30. Laby RJ, Kincaid MS, Kim D, Gibson SI: The Arabidopsis sugar-insensitive mutants sis4 and sis5 are defective in abscisic acid synthesis and response. The Plant Journal 2000, 23(5):587-596.

Page $8 / 13$ 
31. Pourtau N, Marès M, Purdy S, Quentin N, Ruël A, Wingler A: Interactions of abscisic acid and sugar signalling in the regulation of leaf senescence. Planta 2004, 219(5):765-772.

32. Krapp A, Quick WP, Stitt M: Ribulose-1, 5-bisphosphate carboxylase-oxygenase, other Calvin-cycle enzymes, and chlorophyll decrease when glucose is supplied to mature spinach leaves via the transpiration stream. Planta 1991, 186(1):58-69.

33. Hoeberichts FA, Van Doorn WG, Vorst O, Hall RD, Van Wordragen MF: Sucrose prevents up-regulation of senescence-associated genes in carnation petals. Journal of Experimental Botany 2007, 58(11):2873-2885.

34. Mao C, Lu S, Lv B, Zhang B, Shen J, He J, Luo L, Xi D, Chen X, Ming F: A rice NAC transcription factor promotes leaf senescence via ABA biosynthesis. Plant physiology 2017:pp. 00542.02017.

35. Bolouri-Moghaddam MR, Le Roy K, Xiang L, Rolland F, Van den Ende W: Sugar signalling and antioxidant network connections in plant cells. The FEBS Journal 2010, 277(9):2022-2037.

36. Ikegami K, Okamoto M, Seo M, Koshiba T: Activation of abscisic acid biosynthesis in the leaves of Arabidopsis thaliana in response to water deficit. Journal of plant research 2009, 122(2):235.

37. Nambara E, Marion-Poll A: Abscisic acid biosynthesis and catabolism. Annu Rev Plant Biol 2005, 56:165-185.

38. Qiu C, Ethier G, Pepin S, Dubé P, Desjardins Y, Gosselin A: Persistent negative temperature response of mesophyll conductance in red raspberry (Rubus idaeus L.) leaves under both high and low vapour pressure deficits: A role for abscisic acid? Plant, cell \& environment 2017, 40(9):1940-1959.

39. Toh S, Imamura A, Watanabe A, Nakabayashi K, Okamoto M, Jikumaru Y, Hanada A, Aso Y, Ishiyama K, Tamura N: High temperature-induced abscisic acid biosynthesis and its role in the inhibition of gibberellin action in Arabidopsis seeds. Plant physiology 2008, 146(3):1368-1385.

40. Zhu G, Ye N, Zhang J: Glucose-induced delay of seed germination in rice is mediated by the suppression of ABA catabolism rather than an enhancement of ABA biosynthesis. Plant and Cell Physiology 2009, 50(3):644-651.

41. Mega R, Meguro-Maoka A, Endo A, Shimosaka E, Murayama S, Nambara E, Seo M, Kanno Y, Abrams SR, Sato Y: Sustained low abscisic acid levels increase seedling vigor under cold stress in rice (Oryza sativa L.). Scientific reports 2015, 5:13819.

42. Rao MV, Lee H-i, Creelman RA, Mullet JE, Davis KR: Jasmonic acid signaling modulates ozone-induced hypersensitive cell death. The Plant Cell 2000, 12(9):1633-1646.

43. Kojima M, Kamada-Nobusada T, Komatsu H, Takei K, Kuroha T, Mizutani M, Ashikari M, Ueguchi-Tanaka M, Matsuoka M, Suzuki K: Highly sensitive and high-throughput analysis of plant hormones using MS-probe modification and liquid chromatography-tandem mass spectrometry: an application for hormone profiling in Oryza sativa. Plant and Cell Physiology 2009, 50(7):1201-1214.

44. Kakar KU, Nawaz Z, Kakar K, Ali E, Almoneafy AA, Ullah R, Ren X-I, Shu Q-Y: Comprehensive genomic analysis of the CNGC gene family in Brassica oleracea: novel insights into synteny, structures, and transcript profiles. BMC genomics 2017, 18(1):869.

45. Nawaz Z, Kakar KU, Ullah R, Yu S, Zhang J, Shu Q-Y, Ren X-I: Genome-wide identification, evolution and expression analysis of cyclic nucleotide-gated channels in tobacco (Nicotiana tabacum L.). Genomics 2018.

\section{Figures}
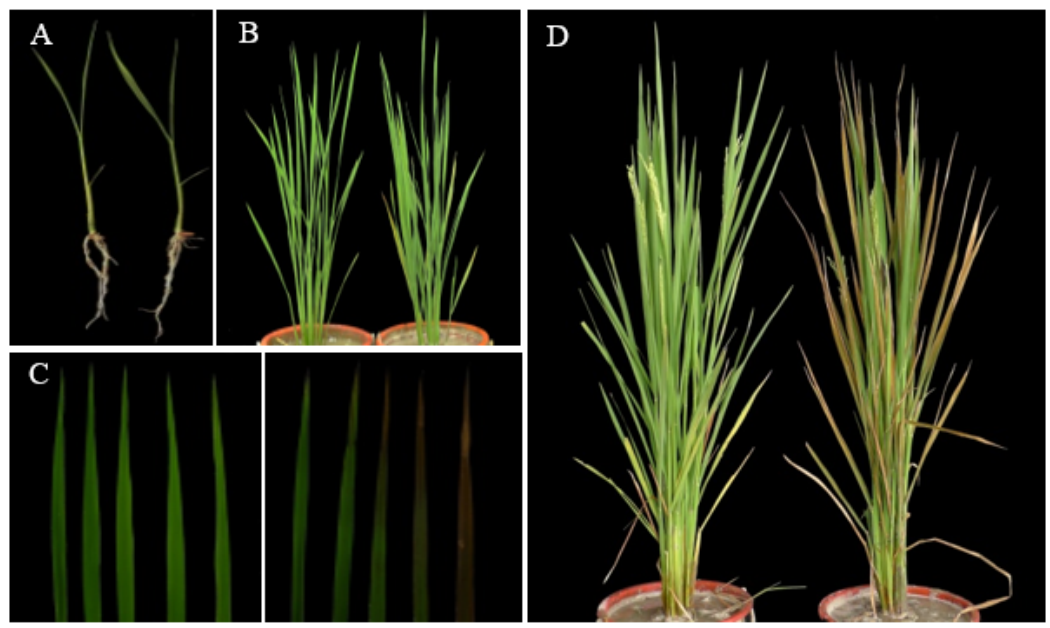

Figure 1

Physiological comparison of early leaf senescence mutant of flag leaves (psf) and its wild at seedling (A), pre antheiss (B), post antheis stages (C) and leaves at post anthesis stage (D). 

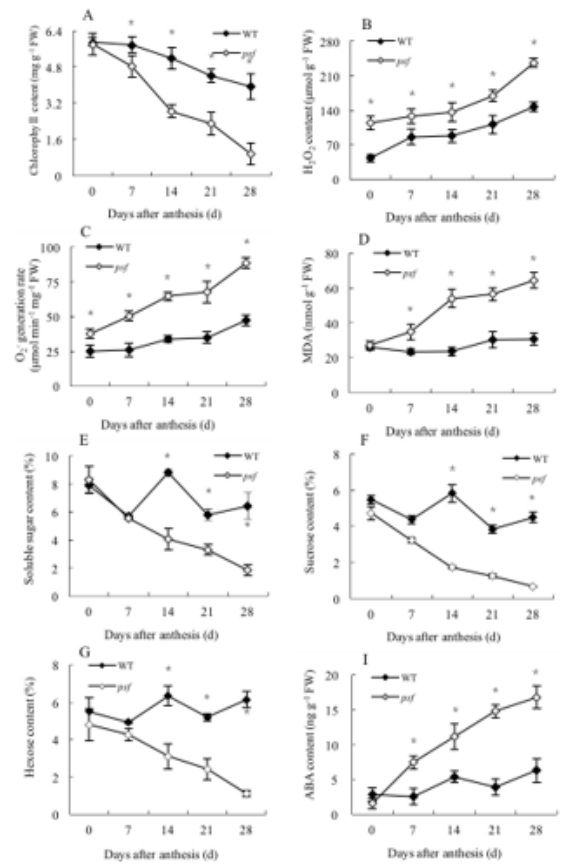

Figure 2

Genotype difference of chlorophyll content, ROS, MDA and sugar content between psf and its wild. Temporal patterns of chlorophyll content (A), H2O2 content (B), 02- generation rate (C), MDA (D), soluble sugar content (E), sucrose content (F), hexose content (G) and ABA content (I) in flag leaves of the psf mutant and its wild type after anthesis. Values are means with the standard errors $(n=3)$. The asterisks represent significant differences $(P<0.05)$ between the psf mutant and its wild type.

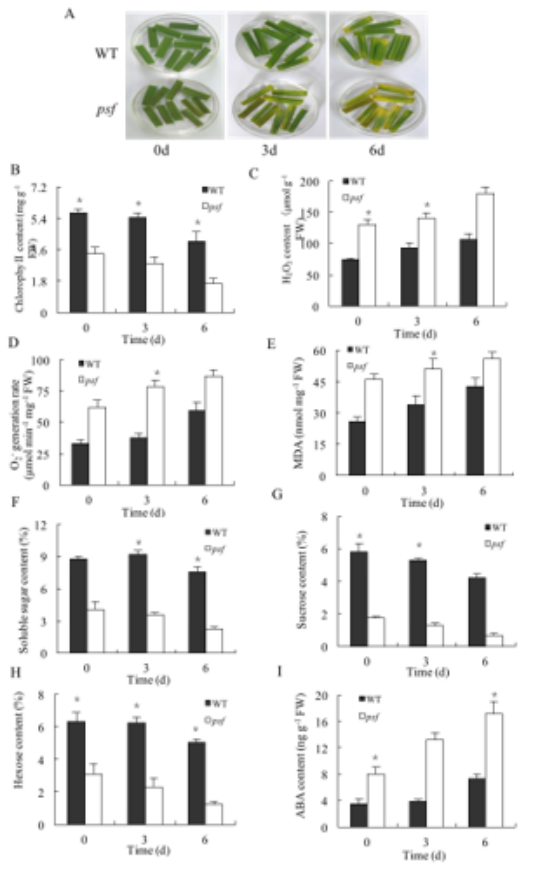

\section{Figure 3}

Senescent symptoms and total chlorophyll content changes of detached leaf segments induced by dark treatment (sugar starvation treatment) after 0,3 and 6 days incubation, respectively. Visual color change of detached leaf segments (A). The chlorophyll contents in detached leaf segments after 0,3 and 6 days incubation (B). Effect of dark treatment (sugar starvation treatment) on $\mathrm{H} 2 \mathrm{O} 2$ content (C), O2- generation rate (D), MDA (E), soluble sugar content ( $F$ ), sucrose content $(G)$, hexose content $(H)$ and $A B A$ content $(I)$ after 0,3 and 6 days incubation, respectively. Vertical bars represent standard deviation $(n=3)$. The asterisks represent significant differences $(P<0.05)$ between the psf mutant and its wild type. 


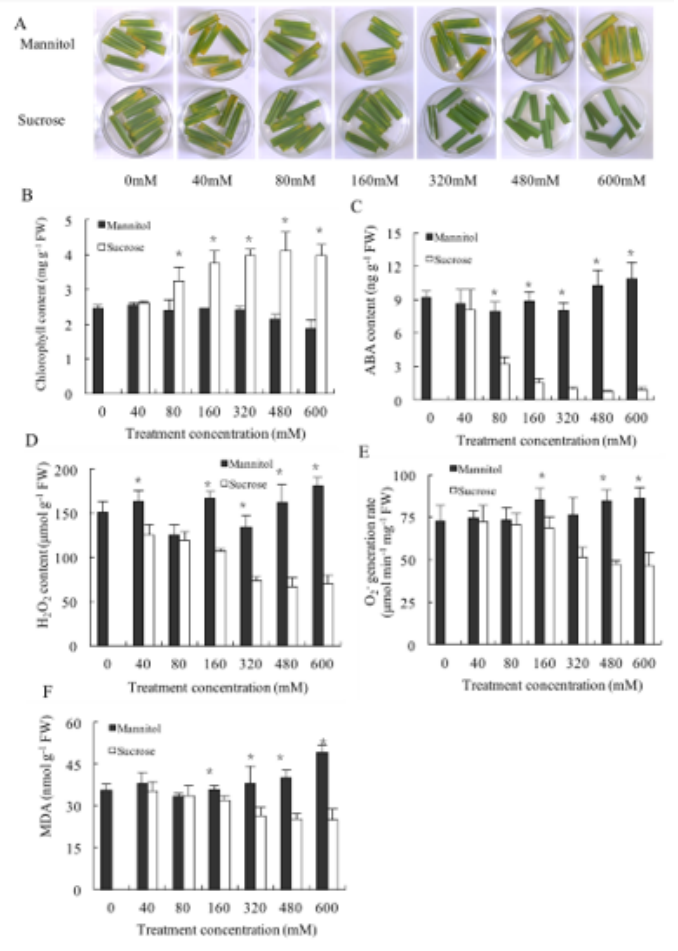

Figure 4

Senescent symptoms, total chlorophyll content changes and its relation to the ABA content of detached leaf segments induced by a gradient concentration of exogenous sucrose and Mannitol ( 0 mM, 40 mM, 80 mM, 160 mM 320 mM, 480 mM and $600 \mathrm{mM})$ after 6 days incubation. Visual color change of detached leaf segments (A). Total chlorophyll contents in detached leaf segments (B), ABA contents in detached leaf (C). H2O2 content (D), 02 - generation rate (E) and MDA (F) at 6 days incubation. Vertical bars represent standard deviation $(n=3)$. * indicates significant differences $(P<0.05)$.

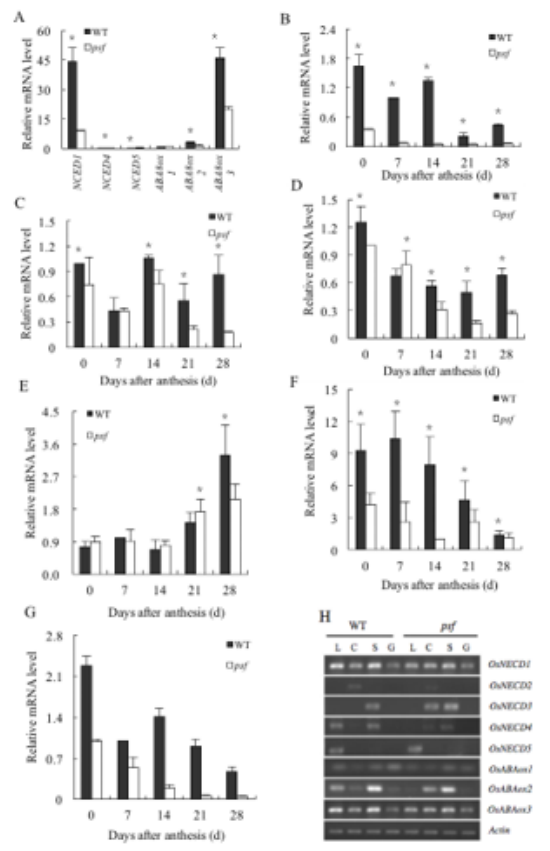

Figure 5

Genotypic difference in the mRNA transcript levels of several key genes involved in ABA synthesis and catabolism and their temporal patterns in the flag leaves of the psf mutant and its wild type after anthesis. Comparison of OsNCEDs and OsABA8oxs isoform genes expressions at 0 day after anthesis (A). Relative mRNA level of OsNCED1 (B), OsNCED4 (C), OsNCED5 (D), OsABA8ox1(E), OsABA8ox2 (F) and OsABA8ox3 (G) Spatial pattern of OsNCEDs and OsABA80xs isoform genes expressions in leaves, culm, sheaths and grains $(H)$. Vertical bars represent standard deviation $(n=3)$. ${ }^{*}$ indicates significant difference $(P<0.05)$. 

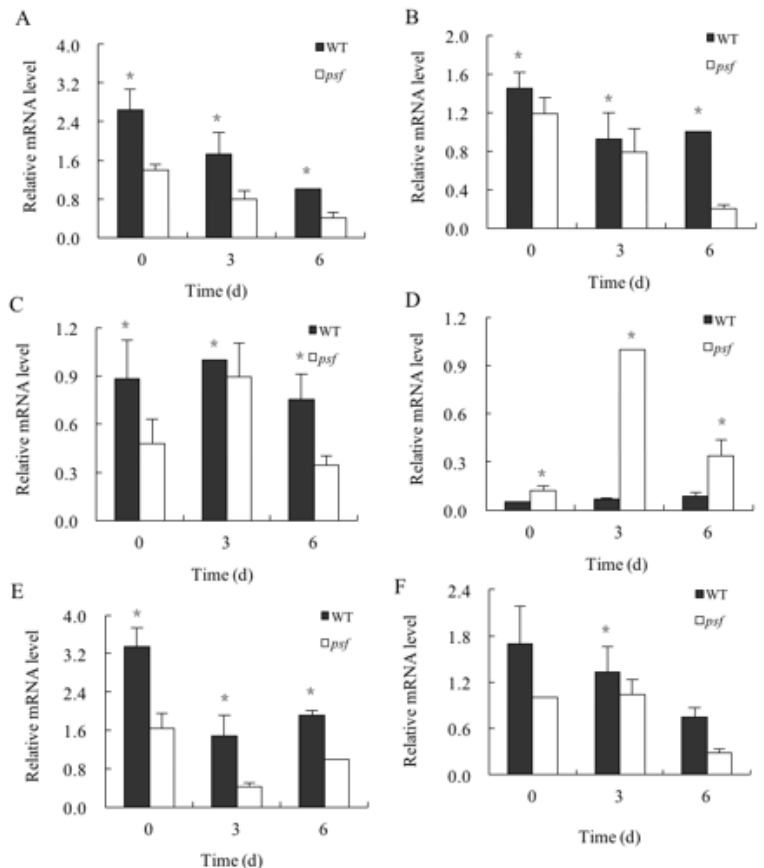

Figure 6

Effects of dark treatment (sugar starvation treatment) on the transcript levels of key genes involved in ABA synthesis and catabolism in leaf segments of the psf mutant and its wild type after 0, 3 and 6 days incubation. Relative mRNA level of OsNCED1 (A), OsNCED4 (B), OsNCED5 (C), OsABA8ox1 (D), OsABA8ox2 $(E)$ and OsABA8ox3 $(F)$. Vertical bars represent standard deviation $(n=3)$. * indicates significant differences $(P<0.05)$.
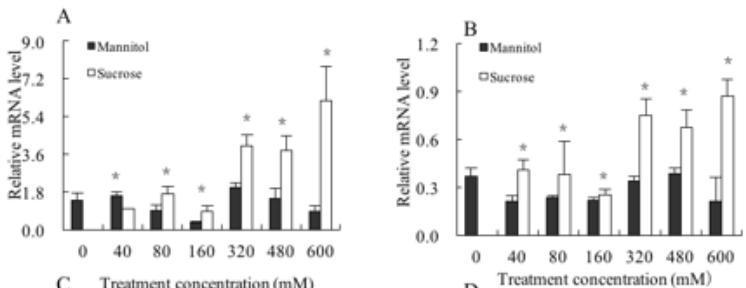

C Treatment concentration $(\mathrm{mM}$

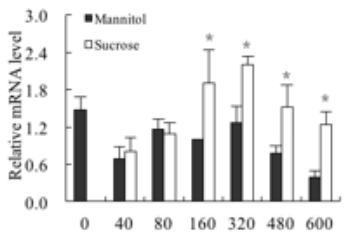

Treatment concentration $(\mathrm{mM})$

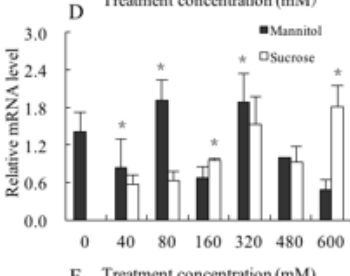

$\mathrm{E}$
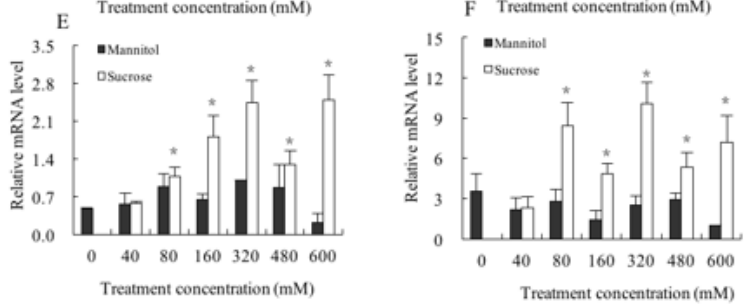

Figure 7

Effect of exogenous gradient concentrations of sucrose treatment on the transcript levels of key genes involved in ABA synthesis and catabolism in the psf mutant and its wild type leaf segments. OsNCED1 (A), OsNCED4 (B), OsNCED5 (C), OsABA8ox1 (D), OsABA8ox2 (E) and OsABA8ox3 (F). Vertical bars represent standard deviation $(n=3)$. * indicates significant differences $(P<0.05)$. 

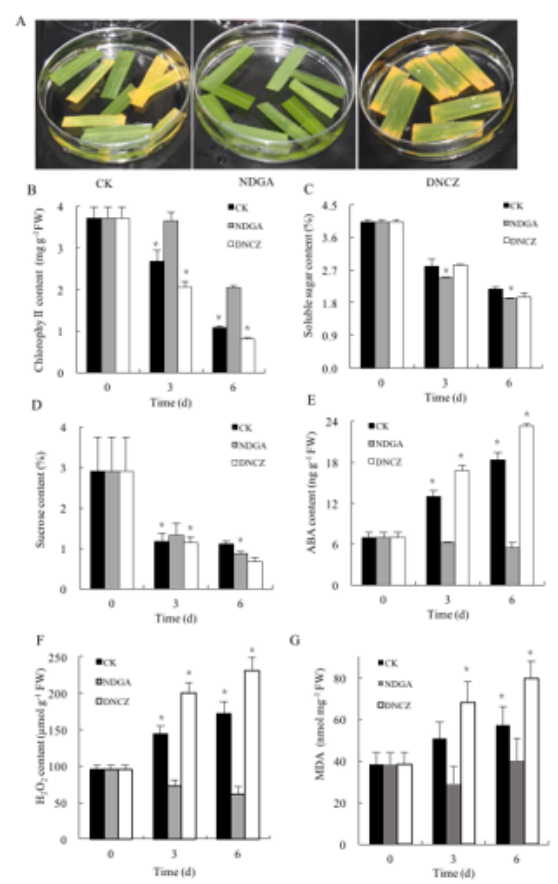

Figure 8

Senescent symptoms, total chlorophyll content, soluble sugar content, sucrose content changes and its relation to the ABA content of detached leaf segments induced by sugar starvation, exogenous NDGA and DNCZ after 0, 3 and 6 days incubation. Visual color change of detached leaf segments after 6 days incubation (A). The chlorophyll contents (B), Soluble sugar contents (C), Sucrose contents (D) ABA contents (E), H2O2 content (F) and MDA (G). Vertical bars represent standard deviation $(n=3)$. * indicates significant differences $(P<0.05)$.

\section{Supplementary Files}

This is a list of supplementary files associated with this preprint. Click to download.

- Supplementarymaterial.docx 